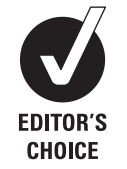

UCT/MRC Research Unit for Exercise Science and Sports Medicine, Department of Human Biology, Sports Science Institute of South Africa, University of Cape Town, Cape Town, South Africa

\section{Correspondence to}

Dr Jeroen Swart, UCT/MRC Research Unit for Exercise Science and Sports Medicine, Department of Human Biology, Sports Science Institute of South Africa, University of Cape Town, PO Box 115, Boundary Road, Newlands, Cape Town 7725

South Africa;

jeroen.swart@uct.ac.za

Accepted 26 August 2011 Published Online First 26 September 2011

\title{
Perceptual cues in the regulation of exercise performance - physical sensations of exercise and awareness of effort interact as separate cues
}

\author{
Jeroen Swart, Timothy Robert Lindsay, Michael lan Lambert, James Craig Brown, \\ Timothy David Noakes
}

\begin{abstract}
It has been argued that the physical sensations induced by exercise, measured as the ratings of perceived exertion (RPE), are distinct from the sense of effort. This study aimed to determine whether a new measure of task effort - the Task Effort and Awareness (TEA) score - is able to measure sensations distinct from those included in the conventional RPE scale. Seven well-trained cyclists completed a maximal effort $100 \mathrm{~km}$ time trial (TT) and a submaximal trial at $70 \%$ of the power sustained during the TT (70\% TT). Five maximal $1 \mathrm{~km}$ sprints were included in both trials. Both the RPE related solely to physical sensation (P-RPE) and the TEA score increased during the TT and were linearly related. During the 70\% TT, both P-RPE and TEA scores increased, but TEA increased significantly less than P-RPE $(p<0.001)$. TEA scores reached maximal values in all $1 \mathrm{~km}$ sprints in both the maximal TT and 70\% TT, whereas the RPE increased progressively, reaching a maximal value only in the final $1 \mathrm{~km}$ sprints in both the TT and the 70\% TT. These results indicate that the physical sensations of effort measured as the P-RPE act as the template regulating performance during exercise and that deviation from that template produces an increase in the sense of effort measured by the TEA score. Together, these controls ensure that the chosen exercise intensity does not threaten bodily homeostasis. Our findings also explain why submaximal exercise conducted within the constraints of the template P-RPE does not produce any conscious awareness of effort.
\end{abstract}

\section{INTRODUCTION}

We and others have provided evidence that a complex, intelligent, central nervous system (CNS) mechanism is responsible for the regulation of exercise performance during endurance exercise of maximal effort. ${ }^{1-5}$ A number of studies have now established that the rate at which perceived exertion increases is a key variable regulating performance during prolonged endurance exercise. ${ }^{6-11}$ Accordingly, Tucker and Noakes have proposed a model in which the ratings of perceived exertion (RPE) are compared with the 'template' of a desired or ideal RPE progression. ${ }^{12} 13$ This template is based on experience and planned in anticipation based on the expected exercise duration. We have recently advanced this concept by showing that certainty about the exercise duration and precise knowledge of the end point of exercise determines the characteristics of this response. ${ }^{5}$

Thus, the rate at which the RPE increases during exercise becomes increasingly linear with both experience and greater certainty about the end point. This finding has been independently confirmed. ${ }^{14}$ The model by Tucker and Noakes proposes that a mismatch between the expected and actual RPE produces an alteration in the workload to correct this mismatch. However, their model does not include a mechanism to explain how the CNS corrects the workload to ensure that the RPE is maintained within the constraints imposed by the predetermined template. Specifically, the model by Tucker and Noakes leaves unanswered the question of whether the decision to modify the workload is determined consciously or subconsciously. This is relevant because the current debate of how the CNS regulates exercise performance focuses on the contrasting views that this controller acts subconsciously ${ }^{15}$ or consciously, ${ }^{16}$ or as a combination of both. ${ }^{17} 18$ De Koning et a ${ }^{19}$ have recently proposed that an index of momentary RPE predicts the subsequent pace chosen and have named this the 'hazard score'. The end point of the event is a key anchor against which the index is measured. However, those authors do not speculate on how the hazard score is perceived within those brain areas responsible for the pacing strategy.

In addition, the exact meaning of the term "perceived exertion' has recently been questioned ${ }^{20}$ on the grounds that the term incorporates both the physical sensations of exercise and the psychological/psychic effort required to perform the task. Borg ${ }^{21}$ acknowledged this in his earlier description of the RPE, "being an expression of the individual's total physical and psychic reaction to exertion." Thus, it has recently been proposed that the physical symptoms induced by exercise are distinct from the sense of effort. ${ }^{17} 20$ We suggest that the sense of effort is a subjective sensation, not based on any (known) physical changes induced by exercise, but which is generated by the brain in response to some as yet unidentified specific component of the exercise bout.

We hypothesise that the mechanisms responsible for the sense of effort - in our terms, the conscious mental (psychic) effort required to sustain or increase the current exercise intensity (work rate) - will be absent when the exercise does not pose a threat to homeostatic control or if the threat is of so small a magnitude that the subject becomes aware of a sense of effort only when specifically questioned by another. Conversely, when the exercise intensity threatens homeostatic control, either imminently or at some time in the future, before 
the completion of the task, this sense of effort will increase in proportion to the magnitude of the imposed threat. This would produce a progressively greater sense of effort, the goal of which is to force the exerciser to reduce the current exercise intensity to ensure that a catastrophic biological failure does not occur during the exercise bout. ${ }^{22} 23$

To investigate the possible independent effects of the physical (RPE) and psychic sensations generated during exercise, we developed a novel perceptual scale to quantify the (psychic) sense of effort, including the individual's conscious awareness of that specific sensation. We have termed this scale the Task Effort and Awareness (TEA) scale.

In addition, the traditional Borg 15 point RPE scale was modified to include only the physical sensations experienced by the subject. We have termed this the physical-RPE (P-RPE) scale. We hypothesised that the rate of progression of the P-RPE would be a key regulator of task effort because, as previously established for the traditional Borg RPE scale, the P-RPE should increase as a generally linear function of exercise duration. Any deviation in the moment-to-moment P-RPE above the brain prescribed RPE template would generate an increased sense of effort measured by an increased TEA score. Ultimately, the increasing sense of effort would generate a conscious signal that would ultimately force the subject to reduce the exercise intensity. In contrast, a P-RPE that increased at a rate slower than that prescribed by the anticipatory RPE template would generate a low TEA score or none at all.

This model therefore attempts to determine why the conscious awareness of the sense of effort occurs. ${ }^{24}$ We hypothesise that the sense of effort plays a role in modifying exercise behaviour specifically to ensure that the P-RPE follows the predetermined template reaching its maximum value only at the point of exercise termination. In particular, we hypothesised that the P-RPE would increase as a linear function of the exercise duration but would not reach maximum values during bouts of intermittent all-out exercise that were interspersed regularly during a more prolonged exercise bout. In contrast, the sense of effort measured as the TEA score would reach maximum values during each maximal effort, indicating that the sense of effort impinges into the subject's consciousness whenever the P-RPE increases above the predetermined anticipatory RPE template for a specific exercise bout.

\section{METHODS \\ Ethical approval}

Before participation, subjects completed the Physical Activity Readiness Questionnaire and were informed of the risks associated with the study. Informed consent was obtained in writing before the initiation of the study. All procedures conformed to the declaration of Helsinki. ${ }^{25}$ The study was approved by the Research and Ethics Committee of the Faculty of Health Sciences of the University of Cape Town Medical School.

\section{Study design}

Seven trained competitive male cyclists were recruited for the study. Subjects reported to the laboratory on four occasions. During the first visit, subjects underwent anthropometric assessment and preliminary testing for measurement of peak oxygen consumption $\left(\mathrm{VO}_{2 \text { peak }}\right)$.

Each cyclist subsequently completed three $100 \mathrm{~km}$ time trials (TTs) that incorporated $5 \times 1 \mathrm{~km}$ sprints at $11,32,52,72$ and $99 \mathrm{~km}$. These trials were completed at weekly intervals.
The first trial acted as a familiarisation TT. Subjects then completed a self-paced maximal $100 \mathrm{~km}$ stochastic TT followed by a submaximal $100 \mathrm{~km}$ TT (70\% TT) trial, which was conducted at $70 \%$ of the average power output achieved during the TT. However, subjects were asked to produce a maximal effort during the $1 \mathrm{~km}$ sprints during both trials. Therefore, in the $70 \% \mathrm{TT}$, the baseline power output was $70 \%$ of the average power sustained during the TT, whereas the sprints were maximal effort in both trials. Each trial was conducted on a simulated flat $100 \mathrm{~km}$ course created on the Computrainer Ergometer (RacerMate, Seattle, USA). Subjects were blinded to all feedback except completed distance during all trials.

During the trials, subjects consumed a commercially available sports drink ( $8 \%$ carbohydrate content) at a rate of 600 $\mathrm{ml} / \mathrm{h}(150 \mathrm{ml}$ every $15 \mathrm{~min})$ because this ingestion rate prevents the development of hypoglycaemia during prolonged exercise. ${ }^{26}$ Additional water was available ad libitum during the trials.

\section{General procedure}

All subjects reported to the cycling laboratory, in which all testing was conducted under stable climatic conditions $(21.4 \pm$ $0.8^{\circ} \mathrm{C}, 46 \pm 3 \%$ relative humidity, $102.2 \pm 0.6 \mathrm{kPa}$ ).

Measurement of body composition, including height, body mass and seven skinfolds (triceps, biceps, suprailiac, subscapular, calf, thigh and abdomen ${ }^{27}$ ), was performed before the start of the initial test $\left(\mathrm{VO}_{2 \text { peak }}\right)$. Each subject's height $(\mathrm{cm})$ and mass $(\mathrm{kg})$ were measured using a precision stadiometer and balance (Model 770; Seca, Bonn, Germany), accurate to $1 \mathrm{~mm}$ and 100 g, respectively.

Body fat content was measured as the sum of seven skinfolds and as a percentage of body mass (\%BF). ${ }^{28}$ Each subject was asked to refrain from consuming any caffeine or other stimulants on the day of each performance test. Before each testing session, subjects were questioned to confirm that they had adhered to these instructions.

All tests were performed on an electronically braked cycle ergometer (Computrainer Pro 3D; RacerMate, Seattle, Washington, USA), which allows subjects to use their own bicycles in the laboratory. The rear wheel tire of each subject's bicycle was inflated to $800 \mathrm{kPa}$, and the bicycle was mounted with a rear axle quick release mechanism. Before the start of the warm-up protocol, the contact pressure of the load generator against the rear wheel was calibrated to $0.88-0.93 \mathrm{~kg}$. After a 15 min self-paced warm-up protocol, the load generator was recalibrated to $0.88-0.93 \mathrm{~kg}$ as recommended. 2930

\section{Perceptual scores}

To measure only the physical sensations experienced during exercise, the traditional Borg 15 point RPE scale was modified to exclude any psychological/psychic contribution to effort perception. We have termed this the physical-RPE (P-RPE) scale because it includes only the physical sensations experienced by the subject.

Before the progressive exercise test, subjects were familiarised with the P-RPE scale according to a standard set of instructions (Appendix B). As described, subjects were asked to consider only the physical sensations of effort and to specifically exclude any psychological/psychic contributions to those sensations.

In addition, subjects were instructed according to a novel scale, which was designed specifically to incorporate two components: (1) the magnitude of the psychological/psychic sensations of effort and (2) the extent to which the subject was consciously 
Table 1 Subject characteristics $(\mathrm{N}=7)$

\begin{tabular}{lc}
\hline Age (years) & $34 \pm 9$ \\
Mass $(\mathrm{kg})$ & $79.5 \pm 13.9$ \\
Height $(\mathrm{cm})$ & $181 \pm 11$ \\
Peak power output $(\mathrm{W})$ & $366 \pm 33$ \\
$\mathrm{VO}_{2 \text { peak }}(\mathrm{ml} / \mathrm{kg} / \mathrm{min})$ & $55.9 \pm 6.2$ \\
Data represented as mean $\pm \mathrm{SD}$ & \\
\hline
\end{tabular}

$\mathrm{VO}_{2 \text { peak' }}$ peak oxygen consumption.

aware of those sensations (TEA scale) (Appendix B). We hypothesised that this sense of effort would increase linearly at a similar rate as the P-RPE during all-out exercise and therefore developed an equivalent 15 point scale. The scale includes negative values to indicate the absence of any sense of effort. The position of the verbal anchors was based on pilot trials.

To confirm that subjects understood the difference between these two scales, each subject completed a 10 point questionnaire after the initial familiarisation with both scales. Subjects were allowed to continue with the trial only if they answered at least nine of the questions correctly.

During each trial, subjects were asked to report their P-RPE and TEA scores verbally and by pointing at a chart after every $5 \mathrm{~km}$ interval and at the end of each $1 \mathrm{~km}$ sprint. During the progressive exercise test, subjects were asked to report their P-RPE and TEA scores at 2 min intervals until exhaustion. Subjects were reminded repeatedly to include only physical sensations for the P-RPE score, whereas for the TEA score, they were asked to consider only the ratings of psychological/ psychic effort.

\section{Progressive exercise test}

Testing for $\mathrm{VO}_{2 \text { peak }}$ was performed at a starting work rate of $100 \mathrm{~W}$. The load was increased incrementally at a rate of 20 W every $60 \mathrm{~s}$ until the subject was unable to sustain a cadence greater than 70 revolutions/min. During the progressive exercise test, ventilation volume $\left(\mathrm{V}_{\mathrm{E}}\right)$, oxygen uptake $\left(\mathrm{VO}_{2}\right)$ and $\mathrm{CO}_{2}$ production $\left(\mathrm{VCO}_{2}\right)$ were averaged every $15 \mathrm{~s}$ using an online breath-by-breath gas analyser and pneumotach (Oxycon; Viasis, Hoechberg, Germany). Calibration of this device was performed before testing according to the manufacturer's instructions. $\mathrm{VO}_{2 \text { peak }}$ was recorded as the highest $\mathrm{VO}_{2}$ measurement recorded for any $30 \mathrm{~s}$ period. Peak power output (PPO) was calculated by averaging the power output for the final minute of the $\mathrm{VO}_{2 \text { peak }}$ test. ${ }^{31}$ Subjects were not allowed to stand on the pedals at any time during the test.

\section{Data collection and analysis}

Power output, speed, cadence and elapsed time were measured during all trials and stored by the Computrainer software at a rate of $34 \mathrm{~Hz}$. Heart rate data were captured with Suunto T6 heart rate monitors (Suunto Oy, Vantaa, Finland) and stored every 2 s. Performance and heart rate data from the PPO test and the $100 \mathrm{~km} \mathrm{TTs} \mathrm{(TT} \mathrm{and} \mathrm{70 \% TT)} \mathrm{were} \mathrm{analysed} \mathrm{for} \mathrm{the} \mathrm{full} \mathrm{period}$ of the data capture and for each $1 \mathrm{~km}$ sprint. Analysis of performance data was performed using CyclingPeaks analysis software (WKO+ edition, Version 2.1, 2006; Lafayette, Colorado, USA). Heart rate data were analysed with the Suunto Training Manager (Version 2.1.0.3; Suunto Oy, Vantaa, Finland).

\section{Statistical analysis}

Data were analysed for statistical significance using STATISTICA version 8.0 (Stat-soft, Tulsa, Oklahoma, USA).

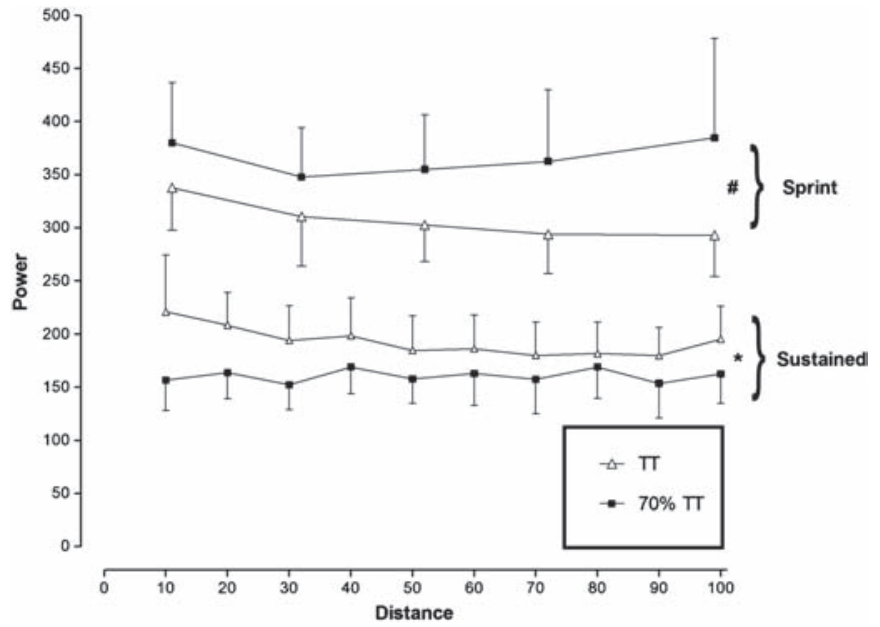

Figure 1 Sustained and sprint power output. * Significant difference between trials for sustained power output $(p=0.05)$. \#Significant difference between trials for sprint power output $(p=0.043)$.

A two-way analysis of variance with repeated measures was used to examine differences between trials. Sphericity of the data was tested using the Mauchley test. ${ }^{32}$ When the sphericity condition was violated, a Greenhouse-Geisser adjustment was made to the degrees of freedom to counter the increased risk of a type I error. Where a significant difference was found for either main effect (trial or time), a Tukey post-hoc analysis was performed. Regression analyses for the relationship between P-RPE and TEA scores were performed for the progressive exercise, both trials (baseline values) and the values recorded during sprints. Differences between the TT and $70 \%$ TT for TEA scores were determined using an unpaired t test.

Statistical significance was accepted when $p<0.05$. All data are expressed as means $\pm \mathrm{SD}(X \pm s)$.

\section{RESULTS}

\section{Subject characteristics}

The descriptive characteristics of the subjects and results for the $\mathrm{VO}_{2 \text { peak }}$ testing are listed in table 1.

\section{Power}

Sustained and sprint power output are shown in figure 1. A significant time $x$ trial interaction was observed for power output $\left(\mathrm{F}_{9,108}=3.76 ; \mathrm{p}=0.0003\right)$. Sustained power output during the $70 \%$ TT was significantly lower than during the TT in accordance with the experimental design (main effect of trial: $\mathrm{F}_{1,12}=4.61 ; \mathrm{p}=0.05$ ). Power output during the $1 \mathrm{~km}$ sprints was significantly greater in the $70 \%$ TT than in the TT (main effect of trial: $\mathrm{F}_{1,12}=5.11 ; \mathrm{p}=0.043$ ).

\section{P-RPE}

P-RPE scores during the continuous section of the trials increased progressively with time in both TT and 70\% TT (main effect of time: $\mathrm{F}_{18.216}=49.5 ; \mathrm{p}<0.001$ ) (figure $2 \mathrm{~A}$ ) and were significantly greater in the TT (mean $=14.4 \pm 0.7)$ than in the $70 \%$ TT (mean $=12.1 \pm 1.2$ ) (main effect of trial: $F_{1,12}=18.6$; $\mathrm{p}<0.001$ ). Sprint $\mathrm{P}-\mathrm{RPE}$ scores (figure $2 \mathrm{~B}$ ) also increased progressively with time in both trials (main effect of time: $\mathrm{F}_{4,48}=33.8$; $\mathrm{p}<0.001)$ and were significantly greater in the TT (mean $=19.1$ $\pm 0.5)$ than in the $70 \%$ TT (mean $=18.3 \pm 0.7)$ (main effect of trial: $\left.\mathrm{F}_{1,12}=5.7 ; \mathrm{p}=0.03\right)$. 
TEA

A significant time $\times$ trial interaction was observed for nonsprint TEA scores $\left(\mathrm{F}_{18,216}=3.62 ; \mathrm{p}<0.001\right)$. TEA scores during the continuous section of the trials increased over time in both the TT and 70\% TT (main effect of time: $\mathrm{F}_{18,216}=23.9 ; \mathrm{p}<0.001$ ) (figure $2 \mathrm{C}$ ) and were significantly greater in the TT (mean = $5.4 \pm 1.4$ ) than in the $70 \%$ TT (mean $=1.4 \pm 1.1$ ) (main effect of trial: $\left.\mathrm{F}_{1,12}=35.2 ; \mathrm{p}<0.001\right)$. However, TEA scores during the sprint component of the trials were not different between TT $($ mean $=9.6 \pm 0.3)$ and $70 \%$ TT $($ mean $=9.7 \pm 0.3)$, nor did they change over time in either group (figure 2D). In both cases, values approached the maximum value of 10.0 at the termination of each individual sprint.

\section{Relationship between P-RPE and TEA scores}

During the progressive exercise test, both P-RPE and TEA scores increased linearly ( $\mathrm{r}=0.93$ for both variables) over time (figure 3). The slope values for P-RPE (slope $=0.84$ ) and TEA (slope = 0.81) did not differ significantly, whereas the Y-intercept values $(\mathrm{P}-\mathrm{RPE}=5.05$ and TEA $=-4.35)$ were similar to the lowest values for both scales.

The relationships between P-RPE and TEA were similar for both the progressive exercise test (slope $=0.94$; Y-intercept $=$ -8.8; $r=0.96$ ) and the TT trial (slope $=0.90 ;$ Y-intercept $=-7.6 ; \mathrm{r}$ $=0.80$ ) (figure 4A,B). However, during the $70 \%$ TT, this relationship changed with lower TEA scores relative to P-RPE (slope = 0.56; Y-intercept $=-6.4 ; \mathrm{r}=0.81$ ) (figure 4C). This was significantly different to the progressive exercise test and TT $(p<0.001)$. The relationship between P-RPE and TEA scores also changed during the $1 \mathrm{~km}$ sprints (slope $=0.11$; Y-intercept $=6.5 ; \mathrm{r}=0.50$ ) (figure 4D) and was significantly different from the values measured during the continuous exercise sections in the TT $(p<0.001)$.

\section{Heart rate}

Mean heart rates were significantly greater during the TT $(142 \pm$ $7 \mathrm{BPM}$ than during the $70 \%$ TT $(130 \pm 5 \mathrm{BPM})(\mathrm{p}=0.004)$.

\section{DISCUSSION}

The first important finding of this study was that subjects were able to differentiate clearly between the physical sensations of exercise and psychological/psychic effort required to produce the required workload, measured by the TEA score. This is clearly seen by the dissociation of these two values when subjects exercised at either a lower exercise intensity in the $70 \%$ TT or during the all-out sprints in both trials.

During progressive exercise to exhaustion for measurement of the $\mathrm{VO}_{2}$ max, the TEA score increased as a linear function of both the exercise intensity (figure 3 ) and the physical sensations experienced during exercise (P-RPE) (figure 4A). The regression lines for these two scales were virtually superimposable. This indicates that the range of the TEA scale is correct; that it has a linear growth function; and that the verbal anchors are correctly positioned.
A

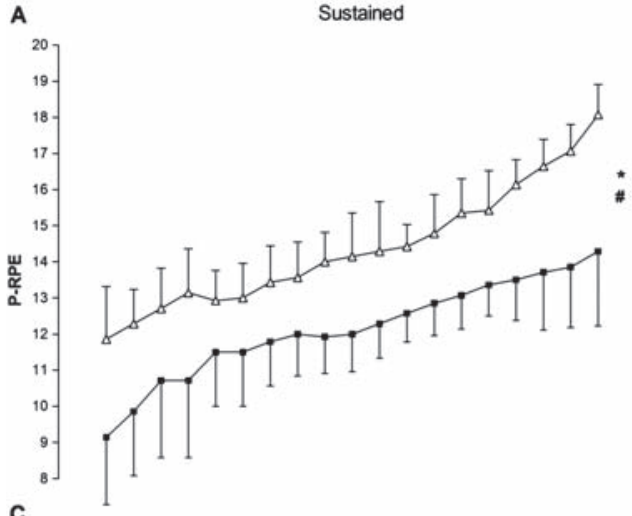

c

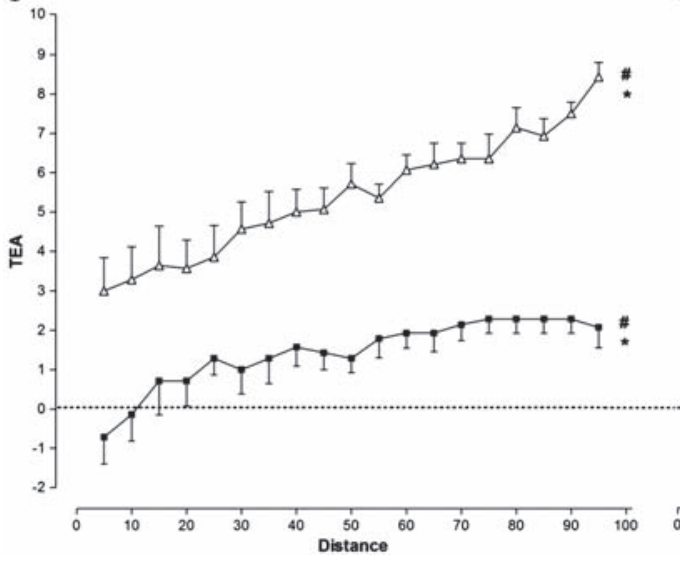

B

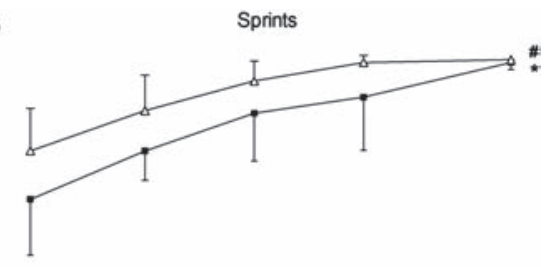

D

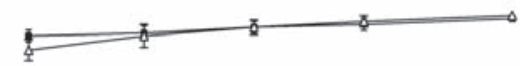

$\approx \pi$

$-70 \% \pi$

Conscious

Subconscious

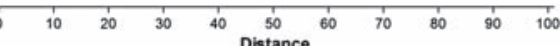

Figure 2 Perceptual scores. (A) RPE scores during continuous exercise. * Significant difference between trials $(p=0.01)$. \#Time main effect, increase over time in RPE in both trials $(p<0.001)$. (B) Sprint RPE scores. ${ }^{*}$ Significant difference between trials $(p=0.02)$. \#Time main effect, increase over time in RPE in both trials $(p<0.001)$. (C) TEA scores during continuous exercise. * Significant difference between trials $(p<0.001)$. \#Time main effect, increase over time in TEA in both trials $(p<0.001)$. (D) Sprint TEA scores. RPE, ratings of perceived exertion; TEA, Task Effort and Awareness. 
In addition, we showed that the perceived exertion scores (P-RPE), modified to include only physical sensations and specifically to exclude sensations relating to psychological/psychic effort, increase progressively and linearly with time during a

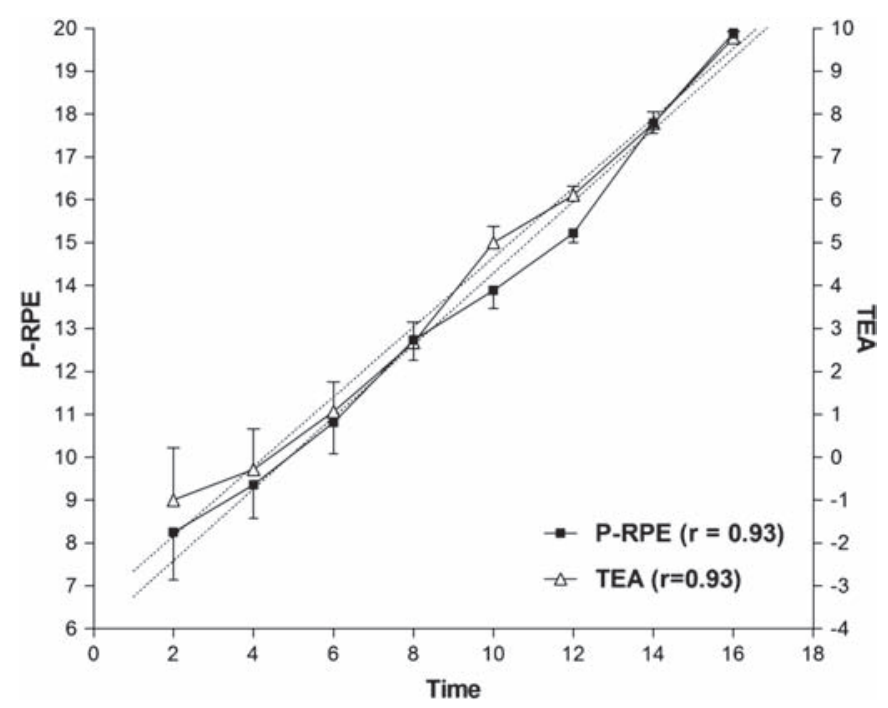

Figure 3 Perceptual scores during a progressive exercise test. Regression lines for TEA and P-RPE do not differ significantly. RPE, ratings of perceived exertion; TEA, Task Effort and Awareness. maximal effort $100 \mathrm{~km}$ cycling TT (figure 2A). Furthermore, the P-RPE values reached near maximal values at or near the end of the exercise bout. This finding supports similar findings in a number of other studies that used the traditional Borg RPE scale. ${ }^{6-10} 33-35$

This finding supports the interpretation that afferent sensory feedback is a key factor regulating exercise performance to ensure that exercise terminates before a catastrophic homeostatic derangement occurs ${ }^{22}$ as opposed to being based purely on feed forward efferent command from the motor cortex, so called 'efference copies'. ${ }^{16}$

To our knowledge, the third important finding is entirely novel. For we have shown that the psychological/psychic effort of exercise, measured by the TEA score, can be dissociated from the physical sensations induced by exercise and are measured by the P-RPE score. On the basis of this finding, we propose that the TEA score measures the psychic effort of maintaining the physical effort that is measured by the P-RPE score.

Thus, we found that both the P-RPE and TEA scores increased over time during exercise of a maximal effort (figure 2A). However, when the exercise was performed at a lower intensity, the P-RPE increased, albeit more slowly (figure 2A - bottom line), whereas the TEA score remained low and within the predominantly subconscious range of the scale (figure $2 \mathrm{C}$ - bottom line). In addition, the TEA scores were significantly lower $(\mathrm{p}<0.001)$ than their respective P-RPE values in comparison to maximal
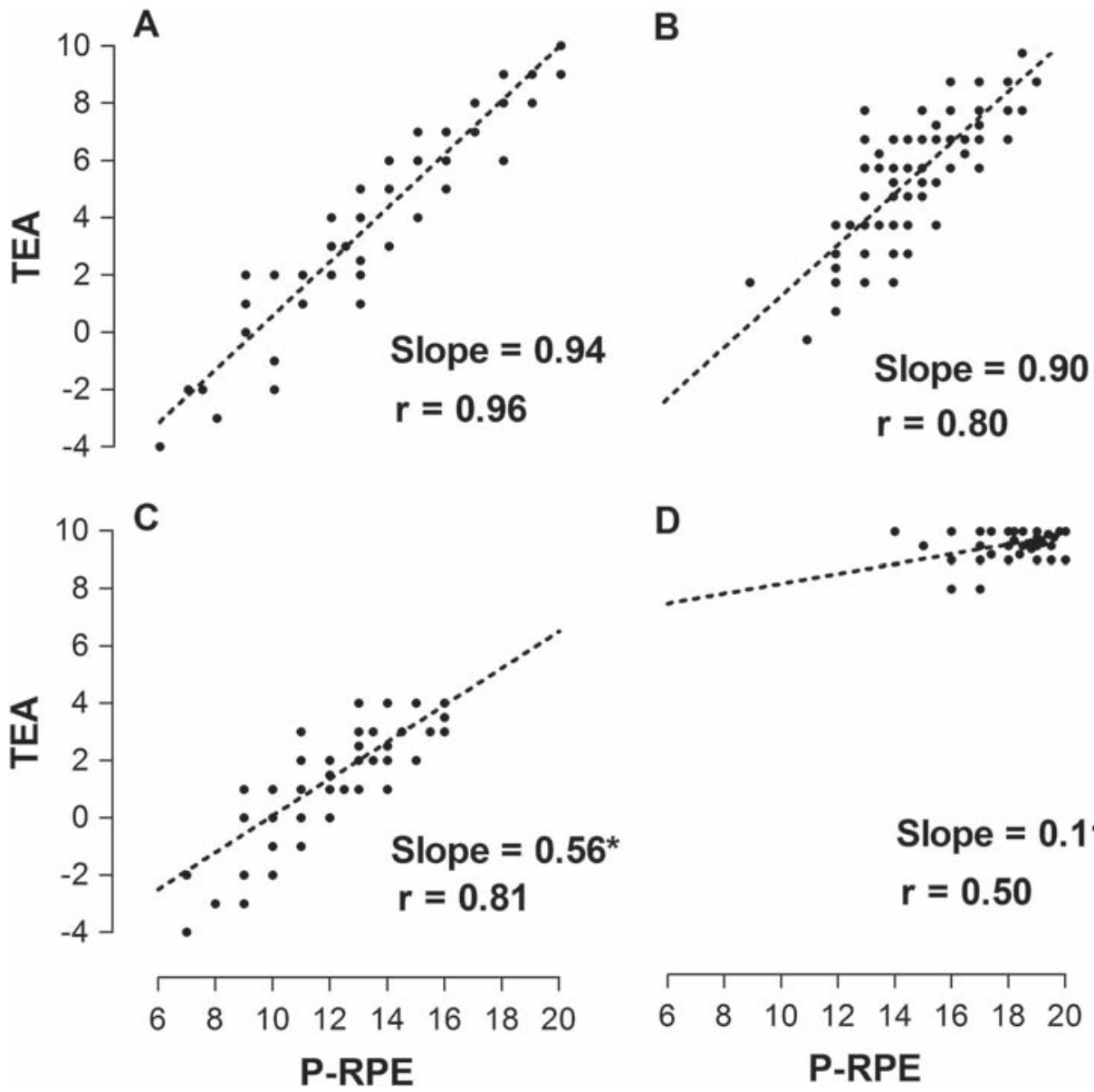

Figure 4 Regression analyses for TEA vs RPE. (A) The progressive exercise test, (B) the time trial (TT), (C) the 70\% TT and (D) sprints from both trials. * Slope and intercepts significantly different from both $A$ and $B(p<0.001)$. ${ }^{*}$ Slope and intercepts significantly different from $A$ and $B$ $(p<0.001)$. RPE, ratings of perceived exertion; TEA, Task Effort and Awareness. 
effort exercise (figure 4C). This shows that the TEA score is not a regulator of exercise performance when the workload is submaximal and that there is no threat to homeostatic control. Under these circumstances, the exercise workload can be maintained with little conscious effort.

In contrast, although P-RPE scores were submaximal (16.219.1) during the $1 \mathrm{~km}$ sprints of both trials (figure $2 B$ ), the TEA scores reached near maximal values for all sprints (9.1-9.9) (figure 2D). TEA scores during sprints were therefore significantly amplified $(p<0.01)$ in relation to their respective P-RPE values compared with the same RPE values recorded during the baseline period of the TT. This finding is also, to our knowledge, completely novel. It appears to show that reaching a maximum sense of effort as measured with TEA scores, and not maximum P-RPE values, is the variable that reflects the CNS process regulating performance during sprints of maximal effort interspersed within a $100 \mathrm{~km}$ TT.

Interestingly, the power outputs achieved during the sprints in the $70 \%$ TT were significantly greater than those achieved during the TT; however, the peak TEA scores were identical. This may indicate that the lower power outputs during the continuous portions of the $70 \%$ TT were associated with a greater homeostatic reserve. As a result, significantly greater power outputs were required during the $1 \mathrm{~km}$ sprints to elicit similar homeostatic disturbances or 'hazard ${ }^{19}$ ' and hence equivalent TEA scores as those reached during the $1 \mathrm{~km}$ sprints during the TT.

These findings are therefore compatible with the following interpretation: the awareness of the sense of effort may therefore represent an emotion derived from a distinct cortical image of homeostatic afferent activity that reflects all aspects of the physiologic condition of all tissues of the body in the context of the remaining work that is required to complete the exercise task. ${ }^{3637}$ Therefore, the conscious decision of whether to maintain, increase or decrease the current workload or indeed to terminate the exercise altogether may be the outcome of a balance between motivation and affect and the sensation that is defined as the sense of effort.

\section{CONCLUSION}

We have provided further evidence that exercise is controlled in a feed-forward and adaptive teleoanticipatory manner. In addition, we have shown perhaps for the first time that the sense of effort and the physical sensations of exercise are distinct but related perceptual cues that play a critical role in the regulation of exercise intensity. Thus, the sense of effort is low during low-intensity exercise, despite progressively increasing physical sensations (measured by P-RPE) as the exercise continues. In contrast, during short sprints of maximal intensity interspersed within the prolonged exercise bout, the sense of effort reaches maximal values, whereas P-RPE values remain submaximal.

These data therefore support the interpretation that exercise is regulated centrally in the brain. ${ }^{12238}$ We propose that this is achieved by altering the rate of progression of perceived exertion via knowledge of the end point and the duration of the exercise bout. ${ }^{511} 1339$ This study shows that if the exercise workload exceeds that required to maintain the predetermined RPE template, a increasing conscious sense of effort as measured by the TEA scale is generated. This may be a conscious representation of the 'hazard score' as hypothesised by de Koning et al. ${ }^{19}$ The direct consequence of the increasing sense of effort will be an altered behaviour, specifically a voluntary reduction in the exercise intensity. Conversely, exercise

\section{What this study adds}

This study adds novel understanding of the pacing mechanisms which occur in the central nervous system during maximal exercise of prolonged duration. A new scale is presented that differentiates task effort from perceived exertion.

intensities that do not pose a threat to homeostatic control produce no or little sense of effort.

Contributors The following authors contributed to data collection, data analysis and writing of the manuscript: JS, TRL, MIL and JCB. TDN contributed to data analysis and writing of the manuscript.

Funding This research was funded by the Medical Research Council of South Africa, the University of Cape Town Harry Crossley and Nellie Atkinson Staff Research Funds, Discovery Health and the National Research Foundation of South Africa through the THRIP initiative.

\section{Competing interests None.}

Ethics approval The study was approved by Research and Ethics Committee of the Faculty of Health Sciences of the University of Cape Town Medical School.

Provenance and peer review Not commissioned; externally peer reviewed.

\section{REFERENCES}

1. St Clair Gibson A, Schabort EJ, Noakes TD. Reduced neuromuscular activity and force generation during prolonged cycling. Am J Physiol Regul Integr Comp Physiol 2001;281:R187-96.

2. Kay D, Marino FE, Cannon J, et al. Evidence for neuromuscular fatigue during high-intensity cycling in warm, humid conditions. Eur J Appl Physiol 2001;84:115-21.

3. Noakes TD, Peltonen JE, Rusko HK. Evidence that a central governor regulates exercise performance during acute hypoxia and hyperoxia. J Exp Biol 2001:204:3225-34

4. Tucker R, Marle T, Lambert EV, et al. The rate of heat storage mediates an anticipatory reduction in exercise intensity during cycling at a fixed rating of perceived exertion. J Physiol (Lond) 2006;574:905-15.

5. Swart J, Lamberts RP, Lambert MI, et al. Exercising with reserve: exercise regulation by perceived exertion in relation to duration of exercise and knowledge of endpoint. Br J Sports Med 2009:43:775-81.

6. Crewe $\mathbf{H}$, Tucker $\mathrm{R}$, Noakes TD. The rate of increase in rating of perceived exertion predicts the duration of exercise to fatigue at a fixed power output in different environmental conditions. Eur J Appl Physiol 2008;103:569-77.

7. Noakes TD. Linear relationship between the perception of effort and the duration of constant load exercise that remains. J Appl Physiol 2004;96:1571-2; author reply $1572-3$.

8. Tucker R, Kayser B, Rae E, et al. Hyperoxia improves $20 \mathrm{~km}$ cycling time trial performance by increasing muscle activation levels while perceived exertion stays the same. Eur J Appl Physiol 2007;101:771-81.

9. Tucker R, Rauch L, Harley YX, et al. Impaired exercise performance in the heat is associated with an anticipatory reduction in skeletal muscle recruitment. Pflugers Arch 2004;448:422-30

10. Marcora SM, Staiano W. The limit to exercise tolerance in humans: mind over muscle? Eur J Appl Physiol 2010;109:763-70.

11. Joseph T, Johnson B, Battista RA, et al. Perception of fatigue during simulated competition. Med Sci Sports Exerc 2008;40:381-6

12. Tucker $\mathbf{R}$, Noakes TD. The physiological regulation of pacing strategy during exercise: a critical review. Br J Sports Med 2009;43:e1.

13. Tucker $\mathbf{R}$. The anticipatory regulation of performance: the physiological basis for pacing strategies and the development of a perception-based model for exercise performance. Br J Sports Med 2009;43:392-400.

14. Foster C, Hendrickson KJ, Peyer K, et al. Pattern of developing the performance template. Br J Sports Med 2009;43:765-9.

15. Amann M, Dempsey JA. Locomotor muscle fatigue modifies central motor drive in healthy humans and imposes a limitation to exercise performance. J Physiol (Lond) 2008;586:161-73.

16. Marcora S. Perception of effort during exercise is independent of afferent feedback from skeletal muscles, heart, and lungs. J Appl Physiol 2009;106:2060-2.

17. Nybo L. Commentaries on viewpoint: perception of effort during exercise is independent of afferent feedback from skeletal muscles, heart, and lungs. J Appl Physiol 2009;106:2064; author reply 2067. 
18. Scano GL. Commentaries on viewpoint: perception of effort during exercise is independent of afferent feedback from skeletal muscles, heart, and lungs. J Appl Physiol 2009;106:2064; author reply 2067.

19. de Koning JJ, Foster C, Bakkum A, et al. Regulation of pacing strategy during athletic competition. PLOS ONE 2011;6:e15863.

20. Smirmaul BD. Sense of effort and other unpleasant sensations during exercise: clarifying concepts and mechanisms. Br J Sports Med 2010;(In Press).

21. Borg G. A simple rating scale for use in physical work tests. Kungliga Fysiografiska Sallskapets I Lund Forhandlinger 1962;32:7-15.

22. Noakes TD, St Clair Gibson A, Lambert EV. From catastrophe to complexity: a novel model of integrative central neural regulation of effort and fatigue during exercise in humans: summary and conclusions. Br J Sports Med 2005;39:120-4.

23. Preston J, Wegener DM. Elbow grease: when action feels like work. In: Morsella E, Bargh JA, Gollwitzer PM, eds. Oxford Handbook of Human Action. Oxford: Oxford University Press 2009:569-86.

24. St Clair Gibson A, Baden DA, Lambert Ml, et al. The conscious perception of the sensation of fatigue. Sports Med 2003;33:167-76

25. Puri KS, Suresh KR, Gogtay NJ, et al. Declaration of Helsinki, 2008: implications for stakeholders in research. J Postgrad Med 2009;55:131-4.

26. Noakes T. Fluid replacement during marathon running. Clin J Sport Med 2003;13:309-18.

27. Ross WD, Marfell-Jones MJ. Kinanthropometry. Editors: MacDougall JD, Wenger HA, Green HS. In: Physiological Testing of the High Performance Athlete. Champaign, IL: 1991:223-308

28. Durnin JV, Womersley J. Body fat assessed from total body density and its estimation from skinfold thickness: measurements on 481 men and women aged from 16 to 72 years. Br J Nutr 1974;32:77-97.
29. Davison RCR, Corbett J, Ansley L. Influence of temperature and protocol on the calibration of the computrainer electromagnetically braked cycling ergometer. J Appl Physiol 2010;10:66-76.

30. Davison RCR, Corbett J, Ansley L. Influence of temperature and protocol on the calibration of the computrainer electromagnetically braked cycling ergometer. J Sports Sci 2007;25:257-8.

31. Lamberts RP, Capostagno B, Swart J, et al. Predicting $\mathrm{VO}_{2 \max }$ and endurance cycling performance from a continuous ramp protocol. Br J Sports Med 2011; (In Press)

32. Winter EM, Eston RG, Lamb KL. Statistical analyses in the physiology of exercise and kinanthropometry. J Sports Sci 2001;19:761-75.

33. Morgan WP, Borg GA. Perception of effort in the prescription of physical activity. In: Craig T, ed. The Humanistic and Mental Health Aspects of Sports, Exercise and Recreation. Chicago, IL: American Medical Association 1976:126-9.

34. Horstman DH, Morgan WP, Cymerman A, et al. Perception of effort during constant work to self-imposed exhaustion. Percept Mot Skills 1979;48:1111-26.

35. Eston R, Faulkner J, St Clair Gibson A, et al. The effect of antecedent fatiguing activity on the relationship between perceived exertion and physiological activity during a constant load exercise task. Psychophysiology 2007;44:779-86.

36. Craig AD. Interoception: the sense of the physiological condition of the body. Curr Opin Neurobiol 2003;13:500-5.

37. Craig AD. How do you feel-now? The anterior insula and human awareness. Nat Rev Neurosci 2009;10:59-70.

38. St Clair Gibson A, Noakes TD. Evidence for complex system integration and dynamic neural regulation of skeletal muscle recruitment during exercise in humans. Br J Sports Med 2004;38:797-806.

39. Noakes TD, Snow RJ, Febbraio MA. Perceived exertion and duration of exercise. J Appl Physio/ 2004;96:1571-3. 